\title{
Perineuritis Successfully Treated with Early Aggressive Immunotherapy
}

\author{
Takumi Nakamura ${ }^{1}$, Takeshi Kawarabayashi ${ }^{1}$, Yusuke Seino ${ }^{1}$, Mie Hirohata ${ }^{1}$, \\ Koichi Wakabayashi ${ }^{2}$ and Mikio Shoji ${ }^{1}$
}

\begin{abstract}
:
Perineuritis is a rare type of peripheral neuropathy defined by swelling and cellular infiltration in the perineurium. We herein report a 52-year-old man who presented with subacute onset pain from the back to the lower limbs, muscle weakness and hypoesthesia. A sural nerve biopsy revealed perineuritis, consisting of inflammatory cell infiltration and swelling of the perineurium. Oral prednisolone, plasma exchange and intravenous immunoglobulin treatment were all effective, leading to significant improvement of the symptoms.
\end{abstract}

Key words: perineuritis, immunotherapy, painful neuropathy, diabetes, rare neuropathy

(Intern Med 58: 2875-2878, 2019)

(DOI: 10.2169/internalmedicine.2638-19)

\section{Introduction}

Perineuritis was first reported in 1972 is as a rare type of peripheral neuropathy characterized by specific pathological findings. Swelling and cellular infiltration in the perineurium, with deposits of immunoglobulin ( $\mathrm{Ig}$ ) G and IgM are the typical pathological findings (1). Comorbidity of diabetes mellitus, leprosy (2), cryoglobulinemia (3), malignancies (including non-Hodgkin's lymphoma) (4-7), ulcerative colitis (8), infection or collagen disease suggests an immune-mediated mechanism (9). Clinical symptoms of perineuritis also vary. The first reported cases were both characterized by an onset with predominantly distal painful sensory neuropathy (1), but cases of onset with mononeuritis multiplex, sensory motor neuropathy or polyradiculopathy have since been reported (8). Therefore, immunosuppressive therapies have historically been employed, and Eric et al. reported that immunosuppressive therapy was effective in 7 out of 12 cases (58\%) of perineuritis (7).

We herein report a case of perineuritis characterized by severe pain in a middle-aged man with well-controlled diabetes that responded well to immunosuppressive therapy.

\section{Case Report}

A 52-year-old man developed back pain 4 months ago and the prick-like pain spread to the entire back. One month later, left foot numbness and foot drop appeared. The same symptoms extended to the right foot, and constipation and nocturia additionally developed. Twenty days before admission to the hospital, right hand numbness and pain in the back and both feet worsened, causing sleeplessness. The administration of analgesics (clonazepam: $2 \mathrm{mg}$, tramadol: 250 $\mathrm{mg}$, amitriptyline: $10 \mathrm{mg}$, alprazolam: $0.4 \mathrm{mg}$, pentazocine injection at $15 \mathrm{mg}$ per day) and a rescue dose of morphine did not improve his pain. He had a few years' history of diabetes mellitus, which was well controlled by dietary therapy, and had drunk approximately $55 \mathrm{~g}$ of alcohol per day over the past 30 years; he stopped drinking at the onset of symptoms.

A neurological examination at admittance revealed distal dominant muscle weakness of both lower extremities, especially of the tibialis anterior (0 in MMT), hypoesthesia below both knees, allodynia in both planta, and loss of lower limb reflexes. He was unable to stand or walk due to painful paraplegia. His blood count was normal. Blood chemistry revealed mild liver damage. Hemoglobin A1c was 6.5\% (ref-

${ }^{1}$ Department of Neurology, Hirosaki University Graduate School of Medicine, Japan and ${ }^{2}$ Department of Neuropathology, Hirosaki University Graduate School of Medicine, Japan

Received: January 8, 2019; Accepted: April 9, 2019; Advance Publication by J-STAGE: June 27, 2019

Correspondence to Dr. Takumi Nakamura, takumi.n@hirosaki-u.ac.jp 
Table. The Results of Nerve Conduction Studies.

\begin{tabular}{|c|c|c|c|c|c|c|}
\hline MOTOR NERVE & \multicolumn{2}{|c|}{$\begin{array}{c}\text { CMAP }(\mathrm{mV}) \\
\text { Distal Proximal }\end{array}$} & \multicolumn{2}{|c|}{$\begin{array}{l}\text { Latency }(\mathrm{msec}) \\
\text { Distal Proximal }\end{array}$} & \multirow{2}{*}{$\begin{array}{l}\mathrm{MCV}(\mathrm{m} / \mathrm{sec}) \\
58.7(>55.0)\end{array}$} & \multirow{2}{*}{$\begin{array}{c}\begin{array}{c}\text { F-latency } \\
(\mathrm{msec})\end{array} \\
27.2\end{array}$} \\
\hline rt. Median & $5.4(>5.0)$ & 4.4 & $3.9(<4.0)$ & 7.7 & & \\
\hline rt. Ulnar & $9.0(>5.0)$ & 3.2 & $2.6(<3.2)$ & 7.6 & $49.5(>55.0)$ & 26.5 \\
\hline rt. Tibial & not evoked & & & & & \\
\hline lt. Median & $8.1(>5.0)$ & 7.6 & $3.5(<4.0)$ & 7.3 & $55.3(>55.0)$ & 27.4 \\
\hline lt. Ulnar & $8.9(>5.0)$ & 6.1 & $2.7(<3.2)$ & 6.9 & $55.4(>55.0)$ & 26.1 \\
\hline lt. Tibial & $0.06(>7.0)$ & 0.05 & $8.4(<5.7)$ & 17.9 & $40.6(>40.0)$ & not evoked \\
\hline SENSORY NERVE & $\begin{array}{r}\operatorname{SNAP}(\mu \\
\text { Distal Prox }\end{array}$ & & $\begin{array}{l}\text { Latency( } \\
\text { Distal Pro }\end{array}$ & $\begin{array}{l}\text { mec) } \\
\text { mal }\end{array}$ & $\begin{array}{c}\mathrm{SCV}(\mathrm{m} / \mathrm{sec}) \\
\text { Distal Proximal }\end{array}$ & \\
\hline rt. Median & $10.4(>14.0)$ & 5.5 & $2.9(<2.9)$ & 6.7 & $52.4(>55.0) 60.5$ & \\
\hline rt. Ulnar & $12.3(>10.0)$ & 6.5 & $2.5(<2.4)$ & 6.4 & $55.6(>55.0) 59.3$ & \\
\hline rt. Sural & $8.1(>8.0)$ & & $1.9(<3.6)$ & & $53.8(>40.0)$ & \\
\hline lt. Median & $5.5(>14.0)$ & 2.3 & $2.9(<2.9)$ & 6.5 & $55.4(>55.0) 54.9$ & \\
\hline lt. Ulnar & $24.1(>10.0)$ & 8.7 & $2.1(<2.4)$ & 5.9 & $65.4(>55.0) 61.2$ & \\
\hline lt. Sural & $4.7(>14.0)$ & & $2.6(<3.6)$ & & $46.9(>40.0)$ & \\
\hline
\end{tabular}
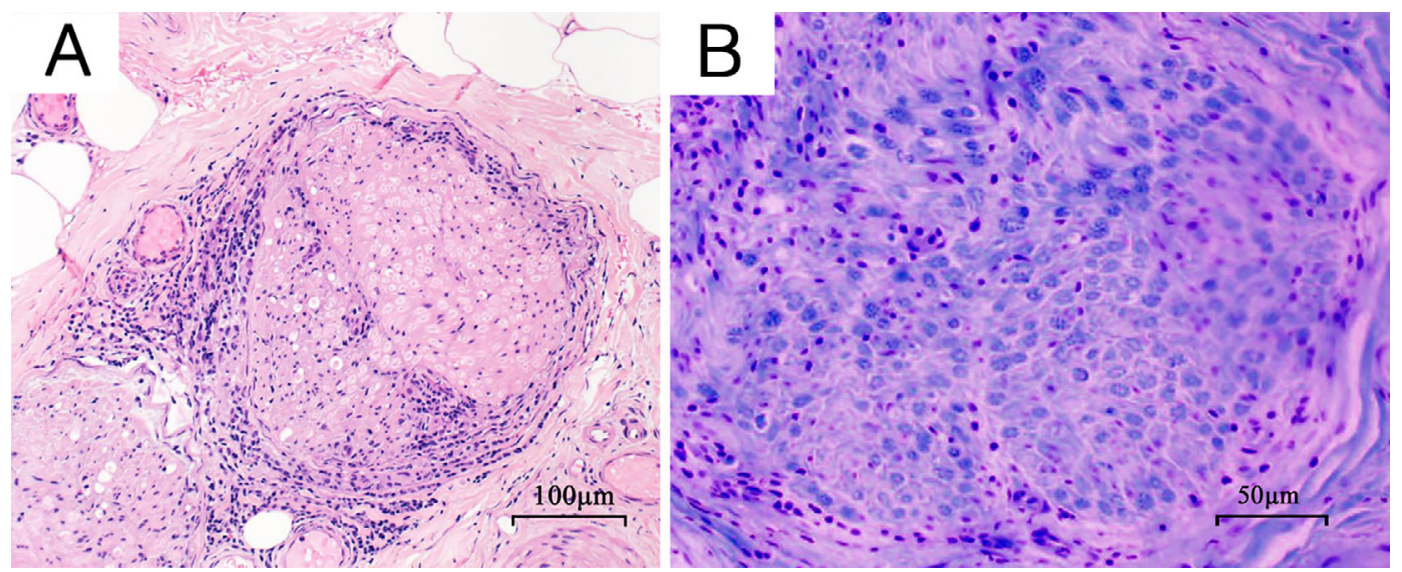

Figure 1. Hematoxylin and Eosin staining (A) and Klüver-Barrera stained (B) images of the sural nerve. Inflammatory cells were distributed in the circumference of the nerve bundle with swelling of the perineurium. Inflammatory cell infiltration in the nerve bundle and loss of myelinated nerve fibers were mild.

erence value: less than 6.2\%), and the fasting blood sugar level was $115 \mathrm{~g} / \mathrm{dL}$. Ferritin was high at $467 \mathrm{ng} / \mathrm{mL}$, but iron and total iron binding capacity were normal. The renal function, thyroid hormone, vitamin B group and blood sedimentation rate were normal. Antinuclear antibody, cryoglobulin and uroporphyrin were negative. A cerebrospinal fluid analysis demonstrated albuminocytologic dissociation with a cell count of $2 / \mu \mathrm{L}$, protein level of $80.1 \mathrm{mg} / \mathrm{dL}$, immunoglobulin $\mathrm{G}$ index of 0.94 , normal myelin basic protein and negative oligoclonal band. Brain and lumbar magnetic resonance imaging findings were normal. Whole-body computed tomography revealed no evidence of malignant tumor. An electromyogram demonstrated denervation and neurogenic changes in the lower limb muscles. Nerve conduction studies indicated decreased compound muscle action potential and sensory nerve action potential with severe motor axon damage to the tibial nerve and mild sensory axon dam- age to the sural nerve (Table). A sural nerve biopsy revealed inflammatory cell infiltration and swelling of the perineurium (Fig. 1). No findings suggestive of vasculitis or abnormal deposits were noted.

Although single oral prednisolone (initial dose of $60 \mathrm{mg}$ per day) therapy slightly improved his pain, the combination of plasma exchange and intravenous immunoglobulin therapy with oral prednisolone markedly improved his muscle weakness and sensory disturbance (Fig. 2). Nearly complete recovery of painful paraplegia, constipation, nocturia and sleeplessness was observed. His condition did not deteriorate following tapering of steroids.

\section{Discussion}

Perineuritis was first reported by Asbury in 1972 as distally dominant recurrent painful neuropathy characterized by 


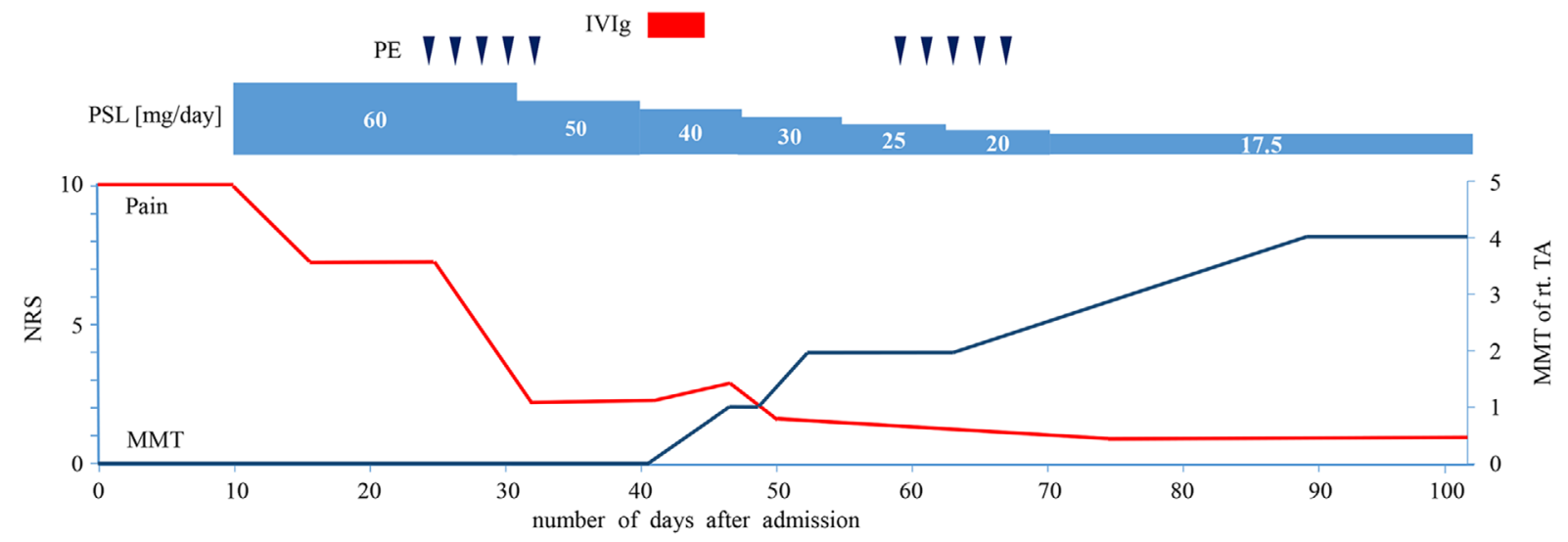

Figure 2. Clinical course after hospital admission. The clinical condition was evaluated based on the degree of pain with the numerical rating scale (NRS) and strength of the tibialis anterior (TA) muscle, which were the most characteristic findings in the present case. Both symptoms improved with treatment. The NRS improved to 1 , and the MMT score for the TA muscle improved to 4 . Prednisone was gradually tapered to $17.5 \mathrm{mg} / \mathrm{day}$ at discharge.

inflammation in the perineurium (1). Other clinical symptoms and comorbid diseases were also reported (1-8, 10). As our patient did not have any other malignancy or autoimmune disease, this case was diagnosed as perineuritis complicated with diabetes mellitus (7).

In this case, the most important differential diagnosis was diabetic neuropathy. Although alcoholic neuropathy was also considered as a differential diagnosis of his painful neuropathy (11), it was excluded because the symptoms were aggravated after temperance in our patient. Some cases of diabetic neuropathy can lead to the subacute onset of painful neuropathy, such as treatment-induced neuropathy of diabetes due to the rapid correction of hyperglycemia (12), acute painful diabetic neuropathy due to the continuation of hyperglycemia (13) or radiculoplexus neuropathy caused by microvasculitis (14) mimicking perineuritis symptoms. Perineuritis is difficult to diagnose by electrophysiological examinations because both axonopathy and demyelination may be observed (7). High levels of protein in the cerebrospinal fluid are not specific among these diseases (7). Perineuritis complicated by diabetes mellitus develops irrespective of blood sugar control (7). Although perineuritis was suggested to be related to diabetes mellitus in previous reports (7), its pathogenesis due to diabetes complications has not been clarified because of the lack of pathologically specific findings. Therefore, a nerve biopsy examination should be performed for diabetes mellitus patients who develop painful neuropathy with no history of rapid correction of hyperglycemia or continuation of hyperglycemia because the treatment approach for perineuritis with diabetes mellitus differs from that for diabetic neuropathy.

Although there is no standard treatment, Asbury first empirically reported on the efficacy of immunosuppressive therapy for the treatment of perineuritis. Eric reported that 7 out of 12 (58 percent) patients with perineuritis saw their condition improved by immunosuppressive therapy including oral or intravenous prednisolone, intravenous immunoglobu- lin, plasmapheresis, immunosuppressant and total lymphoid irradiation (7). In our case, single oral prednisolone administration was not sufficient for relief. Although there was no exacerbation of blood glucose control, no improvement was observed. Therefore, we conducted plasmapheresis and intravenous immunoglobulin administration simultaneously with oral prednisolone. These combined treatments were effective, and the patient had almost no pain or muscle weakness when low doses of analgesics were administered (pregabalin: $150 \mathrm{mg}$ per day and clonazepam: $1 \mathrm{mg}$ per day).

At present, the patient is receiving oral prednisolone according to the standard treatment for vasculitis syndrome. We intend to taper oral prednisolone as much as possible. If recurrence or side effects of prednisolone appear, we will consider the addition of an immunosuppressant. A trial for combined immunotherapy may be useful for establishing a standard therapy for perineuritis with diabetic mellitus.

The authors state that they have no Conflict of Interest (COI).

\section{References}

1. Asbury AK, Picard EH, Baringer JR. Sensory perineuritis. Arch. Neurol 26: 302-312, 1972.

2. Koike H, Hashimoto R, Tomita M, et al. The wide range of clinical manifestations in leprous neuropathy. Intern Med 50: 22232226, 2011.

3. Konishi T, Saida K, Ohnishi A, Nishitani H. Perineuritis in mononeuritis multiplex with cryoglobulinemia. Muscle Nerve 5: 173-177, 1982.

4. Tomita M, Koike H, Kawagashira Y, et al. Clinicopathological features of neuropathy associated with lymphoma. Brain 136: 25632578, 2013.

5. Yamada M, Owada K, Eishi Y, Kato A, Yokota T, Furukawa T. Sensory perineuritis and non-Hodgkin's T-cell lymphoma. Eur Neurol 34: 298-299, 1994.

6. Furusho K, Watanabe M, Ohkoshi N, Tamaoka A, Shoji S. A case of sensory perineuritis with Bowen disease. Rinsho Shinkeigaku 42: 527-529, 2002 (in Japanese, Abstract in English). 
7. Sorenson EJ, Sima AA, Blaivas M, Sawchuk K, Wald JJ. Clinical features of perineuritis. Muscle Nerve 20: 1153-1157, 1997.

8. Chad DA, Smith TW, DeGirolami U, Hammer K. Perineuritis and ulcerative colitis. Neurology 36: 1377-1379, 1986.

9. Bourque CN, Anderson BA, del Campo CM, Sima AA. Sensorimotor perineuritis-an autoimmune disease? Can J Neurol Sci 12: 129-133, 1985.

10. Lee SS, Yoon TY. Sensory perineuritis presented as a mononeuritis multiplex associated with livedo vasculitis. Clin Neurol Neurosurg 103: 56-58, 2001.

11. Koike H, Iijima $M$, Sugiura $M$, et al. Alcoholic neuropathy is clinicopathologically distinct from thiamine-deficiency neuropathy. Ann Neurol 54: 19-29, 2003.
12. Gibbons CH, Freeman R. Treatment-induced neuropathy of diabetes: an acute, iatrogenic complication of diabetes. Brain 138: 4352, 2015.

13. Thomas PK. Classification, differential diagnosis, and staging of diabetic peripheral neuropathy. Diabetes 46: S54-S57, 1997.

14. Dyck PJ, Norell JE, Dyck PJ. Microvasculitis and ischemia in diabetic lumbosacral radiculoplexus neuropathy. Neurology 53: 21132121, 1999.

The Internal Medicine is an Open Access journal distributed under the Creative Commons Attribution-NonCommercial-NoDerivatives 4.0 International License. To view the details of this license, please visit (https://creativecommons.org/licenses/ by-nc-nd/4.0/).

(C) 2019 The Japanese Society of Internal Medicine Intern Med 58: 2875-2878, 2019 